\title{
Horizons/Théâtre
}

Revue d'études théâtrales

10-11 | 2017

Genre et arts vivants

\section{La mise en scène d'une prise de voile : Yvonne Hautin, l'Église et le théâtre}

\section{Henry Phillips}

\section{(2) OpenEdition}

1 Journals

\section{Édition électronique}

URL : http://journals.openedition.org/ht/601

DOI : 10.4000/ht.601

ISSN : 2678-5420

\section{Éditeur}

Presses universitaires de Bordeaux

\section{Édition imprimée}

Date de publication : 1 juillet 2017

Pagination : 296-307

ISSN : 2261-4591

\section{Référence électronique}

Henry Phillips, «La mise en scène d'une prise de voile : Yvonne Hautin, l'Église et le théâtre », Horizons/ Théâtre [En ligne], 10-11 | 2017, mis en ligne le 01 juillet 2018, consulté le 20 juillet 2019. URL : http:// journals.openedition.org/ht/601 ; DOI : 10.4000/ht.601

\section{(c)}

La revue Horizons/Théâtre est mise à disposition selon les termes de la Licence Creative Commons Attribution - Pas d'Utilisation Commerciale - Pas de Modification 4.0 International. 


\section{HeNRY Phillips}

Diplômé d'Oxford, Henry Phillips qui a enseigné aux universités d'Aberdeen (Écosse) et de Cambridge, est professeur émérite de I'Université de Manchester en Grande-Bretagne. Spécialiste des rapports entre l'Église et le milieu théâtral et plus généralement de la culture religieuse au XVII' siècle en France, il s'est aussi intéressé aux liens entre culture et théâtre en France au $x x^{e}$ siècle. II est l'auteur notamment de The Theatre and its Critics in SeventeenthCentury France (Oxford, Oxford University Press, 1980), Church and Culture in SeventeenthCentury France (Cambridge, Cambridge University Press, 1997), et Le Théâtre catholique en France au $x x^{e}$ siècle (Paris, Champion, 2007). Le professeur Phillips a été responsable du classement du fonds Henri Brochet, grande collection de documents importants relatifs au théâtre catholique entre 1922 et 1952, hébergée à la Bibliothèque Jacques-Lacarrière (Auxerre) et accessible en ligne.

Mail : jh.phillips@ntlworld.com

Résumé : En 1932, la prise de voile d'Yvonne Hautin, pensionnaire peu reconnue de la Comédie-Française, au couvent bénédictin de la rue Monsieur à Paris, a suscité l'intérêt de la presse française. La stratégie de l'Église dans l'organisation de cet événement public soulève un certain nombre de questions concernant la place des actrices au sein de la religion. Nous explorons la notion de starisation, pas seulement dans la sphère publique, mais aussi dans la façon dont l'Église s'en empare à cette occasion. Nous retraçons le parcours de vie d'Yvonne Hautin depuis sa décision de quitter la scène pour

Abstract: In 1932, the French press took a particular interest in the ceremony during which Yvonne Hautin, a lowly "pensionnaire" of the Comédie-Française, took the veil as a Benedictine nun, at the convent of the rue Monsieur in Paris. The church's strategy in organising this very public event raises a number of questions concerning the place of the actress in religion. We are led to interrogate the notion of the "star" not only in the public sphere but also as promoted on this occasion by the church. We follow Yvonne Hautin's life from her decision to abandon her career to the ceremony itself. Special la religion jusqu'à sa prise de voile. Nous porterons une attention particulière aux moments où le profane et le sacré semblent entrer en conflit.

Mots-cLÉs : actrice, théâtre, religion, sacré, profane

attention is focussed on those moments where the sacred and the profane seem to clash.

Kerwords: actress, theatre, religion, sacred, profane 


\section{La mise en scène $d$ 'une prise de voile : Yvonne Hautin, l'Église et le théâtre}

En 1930 et en 1932, deux événements ont défrayé la chronique en France (et, soit dit en passant, aux États-Unis) ${ }^{1}$ : le premier, le renoncement d'une pensionnaire de la Comédie-Française, Yvonne Hautin, à sa carrière théâtrale pour devenir religieuse ; le second, sa prise de voile après deux ans de noviciat pendant lesquels, entre autres, elle accompagnait et soignait les malades à Lourdes. La cérémonie a lieu le 17 juin 1932, à Paris, à la chapelle du couvent bénédictin de la rue Monsieur, aujourd'hui disparue. La presse écrite prend un singulier intérêt pour cette intrusion de la religion au sein de la Comédie-Française, la scène la plus prestigieuse de France à l'époque.

Ce n'était pas n'importe quelle cérémonie. En présence de la presque totalité des sociétaires de la Comédie-Française, le cardinal-archevêque de Paris, Monseigneur Verdier, officie avec Monseigneur Gerlier, évêque de Tarbes et Lourdes. Béatrix Dussane, l'une des grandes personnalités de la Comédie-Française, et Madame Émile Fabre, femme de l'administrateur général, remplissent la fonction de « paranymphes » ou de « marraines », choisies par la postulante pour l'accompagner à l'autel. La décoration de la chapelle témoigne d'une somptuosité soignée. Des cartes d'invitation sont distribuées au public et à la presse.

Nous interrogeant dans notre livre, Le Théâtre catholique en France au $\mathrm{XX}^{e}$ siècle $^{2}$, sur les raisons de la faveur extraordinaire accordée à une simple pensionnaire qui n'avait jusqu'alors joué que des rôles de second ordre, nous avons conclu que l'Église avait saisi la cérémonie de la prise de voile comme une deuxième tentative de récupération d'une institution de l'État laïque par l'Église. La première a eu lieu en 1922, lorsque Georges Le Roy, sociétaire de la Comédie-Française, avait, au moment du tricentenaire de la naissance de Molière, organisé en l'église Saint-Roch et avec l'aval du cardinal-archevêque de Paris, la «messe de Molière », que Le Roy nommait aussi des « funérailles nationales $\gg$. Celles-ci visaient à corriger l'erreur de l'Église qui en 1672 avait refusé à Molière les sacrements et la sépulture chrétienne du simple fait de sa profession. L'on doit donc envisager les deux événements dans la 
perspective de possibilités propagandistes directes et indirectes. Notons que Béatrix Dussane et Jeanne Delvair, celle-ci également sociétaire du Français, ont officié en tant que marraines de Simone Suprin, artiste lyrique qui entre chez les Dominicaines en 1930, mais, cette fois-là, tout à fait sans publicité.

Plus généralement, étant donné l'hostilité envers le théâtre véhiculée par l'Église depuis l'époque de saint Augustin et en France jusqu'au début du $\mathrm{XX}^{\mathrm{e}}$ siècle, mais surtout au $\mathrm{XVII}^{\mathrm{e}}$ siècle, cette réorientation d'attitudes passe en particulier par la personne de la comédienne, censée transgresser la modestie qui s'attache proprement à la condition féminine. À travers la prise de voile d'une actrice, l'Église reconnaît que la vie théâtrale ne déniait à aucun de ses praticiens la possibilité d'une vie spirituelle légitime, c'est-à-dire une vie qui va à l'encontre de la seule mondanité. À cet égard, l'Église se rappelait sans doute qu'en 1923, les pompes funèbres de Sarah Bernhardt après la cérémonie en l'église Saint-François-de-Sales, avaient revêtu un caractère exclusivement laïque, dans les rues de Paris bordées de milliers de spectateurs où des personnalités politiques et diplomatiques suivaient le cercueil.

C'est la promotion si publique de la cérémonie de prise de voile qui nous intéresse, surtout en ce qu'elle comportait de grands risques. En effet, l'Église entre dans le monde de la célébrité et s'expose à l'intérêt d'une presse pour laquelle de tels événements alimentent la curiosité des lecteurs et des lectrices. La séduction du public opère à travers la figure de l'actrice. Citons le compte rendu récent du livre Comédienne, de Mademoiselle Mars à Sarah Bernhardt dans le magazine Histoire : «Comédienne... mot de passe pour les rêves de talent et de gloire. Dans l'imaginaire actuel, il se confond volontiers avec star, mot apparu vers le milieu du XIX ${ }^{\mathrm{e}}$ siècle. Se met alors en place un processus de starisation de la comédienne, fascinante à la scène comme à la ville ${ }^{3}$. 》 Ajoutons que la starisation constitue un processus de construction avec ou sans la complicité de la personne concernée.

Parle spectacle quel'Église offre au public et le reportage quil'accompagne $e^{4}$, surtout en ce qui concerne une jeune comédienne, le religieux cède-t-il au mondain, ou serait-ce plutôt le contraire ? L'Église échappe-t-elle au processus de construction que nous venons d'évoquer?

Yvonne Hautin répugnait à toute spéculation sur son renoncement à la scène et ne fournit aucun détail sur les raisons précises de sa décision en dehors de celles qui concernent la seule religion. Dans l'entretien avec Béatrix Dussane qui paraît dans Le Journal, elle affirme être arrivée à sa décision « $[\mathrm{s}]$ ans crise, sans aucun incident extérieur $\gg$. Se demandant pourquoi on s'occupe tant d'elle, elle répond : « C'est une histoire si simple. » (26.09.1930) 
Un article d'Aux Écoutes fait mention d'une vocation subite sans chagrin d'amour ni déception quelconque (27.09.1930), situation confirmée par son frère dans La Dépêche algérienne (19.06.1932).

En fait, elle exprime le souhait à Béatrice Dusssane qu'on l'oublie « et surtout qu'on n'imprime plus mon nom $\gg$. Au cours d'une conversation avec Marcelle Maurette rapportée dans le Journal de la femme, qui a dû avoir lieu entre 1930 et 1932, mais qui paraît bien plus tard, en mars 1934, on apprend que, pour éviter d'être harcelée, « [b]ien conseillée, elle disparut soudain, comme engloutie » et que, après sa dernière représentation au théâtre, « elle sortait sans bruit de la vie ». Marcelle Maurette ajoute : « On l'oublia. » (10.03.1934). Pas exactement.

La presse, pour sa part, trouvait impossible d'en rester là. Après tout, la disparition de la scène d'une comédienne est particulièrement significative dans la mesure où on ne l'entend ni ne la voit plus. Elle abandonne sa vie publique et son public. Cette disparition exige par conséquent une explication.

Cette explication hésite en fait entre l'abandon du futur succès et la médiocrité de sa carrière qui prend donc fin opportunément. Pour L'Ami du Peuple, elle entra au couvent «malgré les promesses d'un talent déjà sûr » (18.06.1932). Le Figaro la décrit comme « une brillante pensionnaire » dont le talent semblait « lui promettre un bel avenir » (18.06.1932). D'autres racontent la déception de sa période au Conservatoire, notamment deux années sans obtenir un premier prix (Régional, Le Mans, 25.09.1930). Sa déprime serait telle que, selon son amie la plus intime, elle pensait se jeter sous une voiture (Aux Écoutes, 27.09.1930), ce qui provoque peutêtre ce cri du cœur de la part d'Yvonne Hautin comme il est rapporté par Marcelle Maurette : « On invente sur moi des choses effrayantes. » (Journal de la femme, 10.03.1934) Dans cette perspective, un article de La Croix des Pyrénées orientales essaie de distinguer l'honnêteté de la personne de sa carrière médiocre : Yvonne Hautin, « simple et modeste », a joué ses rôles secondaires « avec une conscience professionnelle qui la faisait apprécier de tous. $\gg(05.10 .1930)$

Si on peut douter de son véritable talent et donc de l'avenir qui l'attendait, l'explication de sa trajectoire spirituelle semble plus claire. Asté d'Esparbès soutient que la comédienne était « poussée par une irrésistible vocation qui devait venir de loin, car elle était nièce d'archevêque » (Comœdia, 24.09.1930). Hélas, la rumeur de ce destin familial est explicitement démentie par Béatrix Dussane. Le primat en question, Monseigneur Isard, archevêque de Bourges, était simplement ami de la famille, laquelle comptait tout de même deux 
tantes entrées dans les ordres (Le Journal, 26.09.1930). Mais les informations reçues de ses proches décèlent une autre source de confirmation pour la vocation d'Yvonne Hautin. Pendant la tournée qu'elle effectue en Égypte avec la Comédie-Française, un derviche annonce aux camarades d'Yvonne que « avant peu une [des leurs] entrerait au couvent » (Comoedia, 24.09.1930). Yvonne « sentait que son destin allait s'accomplir » (Le Journal, 25.09.1930). Sceptique quant à la sincérité de sa vocation religieuse, Clément Vautel, dans un autre article du Journal de la même date, observe qu'Yvonne Hautin n'a pas encore atteint ses trente ans : « ce n'est pas à cet âge que, d'ordinaire, les femmes, surtout au théâtre, se sentent irrésistiblement entraînées par la vocation monacale $\gg$.

Pourtant, les augures extérieurs cachent selon quelques-uns des sentiments plus profonds, évidents dans son regard. Certes, son « caractère mystique s'accentuait chaque jour » grâce à sa fréquentation des établissements religieux et de son "oncle" l'archevêque $\gg$ (La Croix des Pyrénées orientales, 05.10.1930), mais, dans les couloirs de la Comédie-Française, « [o]n revoyait son clair visage $[\ldots]$ ses grands yeux à la fois enfantins et mystiques $\gg$ ( Le Journal, 25.09.1930). La surenchère du portrait médiatique est à son comble dans un article d'Aux Écoutes : «Une étrange créature. Assez grande, plutôt grasse que maigre, de beaux yeux, la bouche sensuelle. Du mysticisme dans son regard, peut-être surtout quelque chose de trouble et de troublant. $\mathrm{Du}$ mystère sur sa vie, avec une atmosphère du romanesque et de drame. » On va jusqu'à se demander $\mathrm{si}$ 《 une ère de mysticisme » ne déferlerait pas sur la Comédie-Française dans sa totalité (27.09.1930).

Venons-en à la cérémonie de 1932 dont la dissémination médiatique couvre toute la Frances. Elle constitue pour le moins « une intéressante actualité », provoquant «un retentissement considérable » (La Croix de l'Est, Nancy, 03.07.1932). Sans surprise, Georges de la Fourchardière, dans un article de L'Euvre (quotidien de tendance socialiste à l'époque), ne suit pas l'enthousiasme général. Décrivant la prise de voile d'Yvonne Hautin comme « une nouvelle sensationnelle », il s'excuse de la part du journal de l'avoir reléguée en quatrième page, alors que, ailleurs, elle fait la une. Il s'exclame : «Ah! dites-nous que ça n'était pas sérieux !... Dites-nous que c'était du cinéma, et que demain Mlle Yvonne Hautin va créer à la ComédieFrançaise une pièce mystique écrite par un académicien, et dont le lancement devait être assuré par la collaboration du clergé $\gg$ (19.06.1932). Pour lui, ce tapage n'est tout simplement pas à prendre au sérieux. 
Dans cette affaire, la coalition du sacré et du profane offre un curieux mélange des genres, car le fait d'avoir rendu cet événement accessible au grand public lui donne précisément un caractère sensationnel. En somme, la prise de voile fait un tabac. On « joue » à guichets fermés et la salle est comble. La Croix de l'Est (Nancy) nous informe que « Le Tout-Paris mondain s'y est intéressé $\gg(03.07 .1932)$, de sorte qu'«[o]n s'était disputé les cartes d'invitation $»$, ce qui avait pour résultat $\mathrm{d}^{\prime} \ll$ emplir ce calme couvent $[\ldots]$ d'une foule élégante et empressée » (journal non identifié et sans date).

La scène qui se déroule en dehors du couvent le jour de la prise de voile présente des aspects qui sont moins susceptibles de nous surprendre, plus familiers que nous ne le sommes aujourd'hui de ces manifestations d'enthousiasme collectif. La rue Monsieur est inondée d'un « flot impatient » qui « vient battre contre le portail d'entrée qu'on a dû fermer pour empêcher une véritable invasion $\gg$ (Le Journal). Le débordement du service d'ordre (car il y en avait) était tel que le cardinal Verdier a dû attendre pour entrer dans la chapelle (Comøedia). Et qui mentionne-t-on en particulier parmi cette foule ? Citons Astier d'Esparbès, toujours dans Comoedia : « un escadron de dames palpitantes barrait la porte et menaçait de tout démolir si on ne les laissait pas entrer. » C'est le Journal de la femme qui nous informera deux ans plus tard que, dans la cour du couvent, « $[\mathrm{d}]$ es dames en toilette la traversaient, tordant leurs talons sur le pavé » (10.03.1934).

Et à l'intérieur de la chapelle, le caractère sacré de la cérémonie religieuse cède décidément le pas pour une partie du public à d'autres sortes de préoccupations. Le Journal observe que « l'assistance offre un curieux mélange de personnalités du monde ecclésiastique et du monde artistique », et Comødia d'ajouter que « les profanes se montraient des célébrités de la Comédie-Française ». En fait, la présence des célébrités féminines en particulier provoque des considérations profanes, même chez un journaliste qui semblait bien comprendre le fond religieux de l'affaire : Asté d'Esparbès nous apprend que Cécile Sorel (personnage voyant s'il en fut) « conte à notre confrère Édouard Helbey quelques souvenirs des plus piquants sur les Mémoires qu'elle prépare... » (Comœdia). L'ordre n'y est pas plus respecté. Pendant la cérémonie même, une demi-douzaine de spectatrices « logée dans l'étroite petite chapelle latérale [...] ont gagné, pour mieux voir, la hauteur d'une marche $\gg($ Excelsior $)$. Pire, quelques femmes, dans la même intention, sont montées sur les chaises: « De temps en temps, un déclic annonce qu'une photographie a été prise. $\gg$ (Comødia) 
L'opposition du sacré et du profane se prolonge de deux façons supplémentaires, notamment à travers le décor et la mode.

Nous sommes, il est vrai, bien à l'église dont la tradition n'a jamais craint le spectaculaire. Pourtant, les récits de la cérémonie évoquent un spectacle qui n'en conforte pas toujours les intentions spirituelles car, selon Asté d'Esparbès, « les parfums profanes se mêlent aux senteurs légères des roses blanches $\gg$ (Comcedia). De surcroit, le lieu saint se transforme en salle de spectacle où « l'ancienne actrice fait son entrée » (La Croix de l'Est, Nancy). L'image de la scène possède aussi son côté cour et son côté jardin (deux termes scénographiques provenant de l'emplacement de la Comédie-Française en 1770 entre le Louvre et le palais des Tuileries), ici presque littéralement : «Dans la petite cour [du couvent] deux cents personnes se pressent. Tout le monde veut jeter un coup d'œil sur l'autre cour - la cour mystérieuse derrière la porte qui ne s'ouvre qu'à chaque prise de voile. » (Comœdia) On évoque aussi le hors scène : la cérémonie avec les religieuses « se passe dans le domaine de la vie monastique, au-delà de la vie parisienne, du monde des spectateurs - un monde où des yeux pleurent quand le spectacle est émouvant » (Excelsior). L'Euvre observe que les paranymphes ont été recrutées « dans des coulisses dont l'atmosphère n'a rien d'une sacristie » (19.06.1932). Une référence peut-être apocryphe en 1975 : Béatrix Dussane, que « les fastes de la cérémonie avaient époustouflée », aurait chuchoté à Yvonne : « Quelle mise en scène! » (Paris-Normandie, 28.05.1975).

De même que pour le lieu, la mode revêtait un aspect tant réel que symbolique, car, dans les premières décennies du $\mathrm{xx}^{\mathrm{e}}$ siècle, les comédiennes du boulevard devaient acheter elles-mêmes leurs costumes, souvent pour deux mises en scène à la fois. Il arrivait que les comédiennes aient recours à des « mécènes » qui leur fournissaient les moyens nécessaires à leurs besoins vestimentaires, leurs costumes étant alors inspectés au cours de ce qu'on appelait la répétition couturière. Dans cette perspective, les articles de presse n'oublient pas de détailler la toilette des assistantes, surtout dans ses contrastes. Alors que « presque toutes les sociétaires avaient arboré des toilettes "noir et blanc", tout à fait de circonstance » (on ne fait attention qu'aux femmes), l'exception est notée : Gabrielle Robinne était « vêtue de bleue (sic) comme un beau ciel d'été » (Comodia)

En toute logique, on attribue une importance particulière aux vêtements de la future religieuse. Elle fait sensation dans sa « somptueuse toilette de mariée », voilée comme elle est de tulle et « couronnée de fleurs » (L'Intransigeant). Elle est charmante dans « sa robe de mariée $»$, ce qui 
provoque un « frisson d'intense émotion $\gg$. On note que ses camarades de la Comédie-Française « se sont fait une joie » de la lui offrir ( $L a$ Croix de l'Est, Nancy, 03.07.1932). L'Euvre est à la hauteur : « Par quel fâcheux oubli n'a-ton pas mentionné sur les programmes le nom du couturier ? » (19.06.1932)

L'accent mis sur cette toilette permet alors le contraste dramatique avec son apparition dans la « robe de bure » d'une bénédictine, cette « sombre robe de pénitence $\gg($ L'Ami du peuple). Tous les récits s'arrêtent longuement sur le processus de vêtir Yvonne de la ceinture, du scapulaire et du voile blanc. Marie Maurette nous montre pourtant une Yvonne Hautin toujours susceptible de réflexes mondains, même devant la perspective de porter pour toujours un habit de religieuse. Au cours de son entretien avec la pensionnaire de la Comédie-Française, « toute rose sous sa pèlerine vieillotte, le bonnet noir ruché des postulantes $\gg$, Yvonne l'interrompt pour lui poser une question sur la forme de manteau que portait la journaliste : « Soyez gentille, levez-vous, que je voie la mode... C'est chic, ce noir, mais le noir est toujours chic... » (10.03.1934)

Si la robe de bure constitue un adieu à la femme mondaine, la coupure des cheveux renforce le départ du milieu de la scène qu'Yvonne Hautin habitait naguère (le reportage du journal américain The Milwaukee Sentinel (23.07.1932), sur l'entrée de Suzanne Delorme dans l'ordre bénédictin, souligne exactement la même chose). Ce moment de la prise de voile a saisi toute l'attention de la presse jusqu'à devenir obsession et à fournir un supplément de sensationnel au reportage. Le titre d'un des articles d'Asté d'Esparbès proclame : « Devant la Comédie-Française tout entière présente, le cardinal Verdier a coupé les cheveux d'Yvonne Hautin hier comédienne » (Comoedia). Les accessoires de la cérémonie comprennent une paire de ciseaux et un linge sur lequel «la chevelure aux mèches brunes tomba » (L’Ami du Peuple). Plus dramatique encore, ses marraines présentent les cheveux d'Yvonne aux ciseaux du sacrifice (Annales politiques et littéraires, 01.07.32), le sacrifice, évidemment, de sa carrière. Pour le journaliste d'Ève, « ce sont les toisons de toutes les héroïnes incarnées par l'artiste ». C'est l'instant parmi les assistants d'une grande émotion, de larmes, où « les visages se crispèrent » (Le Figaro). Même la main du cardinal « tremble un peu $\gg$ (Comodia). Ce qui provoque le frisson, c'est le crissement de l'acier qui fait redoubler l'émotion : « Tout le monde est avide de voir. » Mary Bell, autre grande dame du Français, « sanglote un peu » (Comøedia).

$\mathrm{Si}$, selon Roger Valbelle d'Excelsior, la cérémonie représente « les adieux à la vie mondaine », la vie mondaine ne laisse pas partir Yvonne Hautin 
sans un brin d'ironie. Jules Véran évoque un dîner en ville où deux femmes « charmantes » estiment que la prise de vêture constitue « un excellent sujet de conversation ». Par ailleurs, nous apprend Véran, « On se chamaille même un peu, partout où on en parle, à cause du caractère, comment fautil dire ? un peu voyant que prit la cérémonie ». Pour l'une des dames, ce qui l'a fait sourire en particulier est justement le frisson qui a accompagné la coupure des cheveux : «Voyons, chère amie ! Autrefois, oui, j'aurais compris ce frisson. Mais aujourd'hui que toutes les femmes se font couper les cheveux! Quelle est celle des artistes de la Comédie-Française qui n’avait pas elle-même subi cette opération ? » Elle banalise la prise de voile en la trouvant $\ll[\mathrm{u}] \mathrm{n}$ joli chapitre à ajouter à l'histoire des rapports de l'Église avec les comédiens $\gg$. Elle suggère même qu'Yvonne Hautin a rendu service aux comédiennes surtout dans la mesure où sa décision demeure heureusement exceptionnelle : «la pauvre petite signait un engagement dont [les artistes féminines] n'étaient pas jalouses. » (Journal non identifié, sans date) Si tout le reportage de la prise de voile honore Yvonne Hautin dans sa personne, l'image générale que l'on présente des femmes ne dépasse jamais le niveau du stéréotype.

L'initiative adoptée par l'Église autour d'Yvonne Hautin possédait donc un aspect paradoxal dans la mesure où elle repose sur le renoncement d'une carrière théâtrale en échange d'une vie en dehors du monde. Si l'Église souhaite bien établir son influence spirituelle au sein de la ComédieFrançaise, a-t-elle choisi la bonne voie ? Clément Vautel le conteste : « Point n'est besoin de se faire nonne ou nonnette pour être vraiment bonne, humble charitable, vertueuse... Je dirai même que ces vertus-là sont plus admirables, plus méritoires, à la Comédie-Française que chez les Bénédictines ! » (25.09.1930) Par contre, l'importance accordée aux sociétaires féminines, participantes ou spectatrices de la cérémonie, compense le départ d’Yvonne Hautin comédienne.

Dans ces circonstances, il faut revenir à la notion de starisation, l'un des éléments qui a motivé cette analyse sur la prise de voile d'Yvonne Hautin. Car force est de poser la question sur les raisons d'une cérémonie si « voyante ». Si on fait abstraction du ton et de l'expression de Georges de la Fourchardière dans l'Euvre, exagère-t-il en déclarant que : «C'était une inconnue, une oubliée qui devait prendre place parmi les sœurs voilées, après avoir été sacrifiée au Seigneur par un simple prêtre dans l'ombre du monastère »? Exagère-t-il outre mesure en se plaignant de ce que « les cabotins du théâtre, $\mathrm{du}$ monde et du journalisme [...] firent des rites assurément respectables une parodie sacrilège $\gg(19.06 .1932)$ ? Il est après tout difficile de concilier 
la grandeur de cet événement avec l'humble statut de son sujet. Alors qu'au moment de sa décision, les reportages insistent tous sur le fait qu'Yvonne Hautin se comporte avec une dignité totale, elle reste bien complice de cet événement sans aucun précédent.

Encore un paradoxe, les comédiennes-sociétaires sont avant tout spectatrices - pour Georges de la Fourchardière dans L'Euvre (19.06.1932) et réduites « à l'emploi de figurantes »-parce que c'est Yvonne Hautin qui constitue le centre d'attention. Ou bien était-ce l'intention de tourner le regard sur toutes les assistantes plutôt que vers la personne d'Yvonne ? Aurait-elle fourni le prétexte de ce spectacle peuplé par les grandes célébrités du théâtre national ?

En réalité, Yvonne Hautin elle-même acquiert le statut qu'elle n'a pas réussi à atteindre au théâtre, précisément celui d'une star. C'est en fait l'Église qui ouvre la possibilité de cette starisation propre au théâtre et qui la consacre par l'occasion de la prise de voile. Mieux, la cérémonie réalise la starisation de la religieuse qu'elle devient : l'Église « construit » un instrument à des fins qui dépassent la personne et la femme qu'est Yvonne Hautin. Georges de la Fourchardière ne se trompe pas : « elle se voit brusquement au premier plan, incarnant un grand premier rôle dans un décor lumineux. » La discrétion qu'a entretenue Yvonne avant la cérémonie devient, par l'entremise de l'Église, indiscrétion.

Tout compte fait, c'est une situation qui déborde manifestement les intentions de l'Église, car celle-ci n'anticipe pas la perception de l'événement par la presse, ni les scènes scandaleuses provoquées par la cérémonie du 17 juin. Pourtant, la presse, à quelques exceptions près, ne se montre pas hostile à la décision d'Yvonne Hautin et de ses suites personnelles sur le plan religieux. En même temps, la presse ne peut s'empêcher de s'intéresser à ce qu'on appellerait de nos jours l'aspect « people » de l'affaire. L'appel au public et aux lecteurs ne se cantonne pas à un intérêt pour la seule religion. Accessoirement, on n'anticipe pas non plus l'irruption de la matérialité indocile dans le processus spirituel de la cérémonie. D’après les récits, le public profane ne perçoit aucun élément de transcendance dans la coupure des cheveux. Elle provoque l'émotion et l'éclat d'un instant spectaculaire digne d'une belle photo. En somme, nous estimons que la stratégie de l'Église a abouti à une catastrophe totale, car le mondain l'a emporté. L'Église s'en rend compte. Les vœux définitifs de l'ancienne pensionnaire se dérouleront en 1934 dans la plus grande discrétion. 
Mais l'histoire d'Yvonne Hautin ne s'arrête pas là. En 1932, sur la jeune moniale, «s'est refermée la porte du cloître » (Le Journal). Elle « se referme sur elle... pour toujours » (Comcdia). Eh bien, non.

Commeun premierpas, Mère Marie-Yvonne, maintenantsupérieure, revient en 1965 à l'attention publique avec la publication d'un livre intitulé Édouard Herriot et Dieu. ${ }^{6}$. Cet ancien président du conseil a soutenu financièrement Yvonne Hautin pendant ses années au Conservatoire, prenant un intérêt actif pour celles qui n'avaient pas les moyens de poursuivre de telles études. Il a aussi fourni le tiers de la dot pour son entrée chez les Bénédictines. Suit en 1969 un deuxième livre, toujours au sujet de Herriot, Lettres à un mécréant ${ }^{7}$, qui, selon une source non identifiée, raconte leur « amitié spirituelle ».

En 1972, elle sort de son couvent, paraissant à la télévision avec Jacques Chancel dans une émission du «Grand Échiquier ». Bien sûr, sa vie de comédienne ne manque pas d'intérêt, mais La France catholique nous informe qu'elle ne souhaitait parler que de la vie religieuse (21.01.1972). On lui pose quelques questions sur sa carrière théâtrale avant qu'elle n'insiste : «Et maintenant, $[\ldots]$ parlons de choses sérieuses. $\gg$ Pourtant, à la demande de Chancel, elle retourne à la Comédie-Française. Ses supérieurs ne lui font aucune objection. Elle a pour seule restriction de ne pas se faire photographier devant l'entrée « parce que cela ferait un attroupement » (La Nouvelle Cité, mai 1972).

L’apogée de sa vie médiatique vient avec sa participation, le 28 mars 1975, au programme légendaire de Bernard Pivot, « Apostrophes » qui s'adressait au sujet, « Jésus-Christ en $1975^{8} \gg$.

Jusqu’à quel point les choses avaient-elles changé ? Les récits de la prise de voile en 1932 nous montrent Yvonne Hautin confiante en elle-même pendant la cérémonie. On la voit « d'une beauté poignante par l'expression de sérénité répandue sur son visage un peu tendu » (Le Journal). Elle reparaît dans sa robe noire : «Souriante, l'air radieux sous sa cornette blanche, avec une aisance et un charme incomparables, elle serre des mains. » (Le Figaro) D'un autre côté, elle demeure un personnage installé dans la grandeur d'une mise en scène exposée au regard public. On ne saurait éviter la conclusion qu'elle reste l'instrument d'une certaine politique. En 1972, l'attention porte cette fois sur ses propres initiatives, c'est-à-dire ses activités d'autrice.

Pourtant, la mise en scène la poursuit en quelque sorte. Le thème de l'absence, si fortement marqué à sa disparition en 1930 pour soigner les malades de Lourdes et à sa sortie du monde en 1932 après la prise de voile, demeure. En effet, une partie de l'émission de Chancel est supprimée, pour 
des raisons inconnues. Elle aurait consisté en une conversation avec Madame Simone, aussi du Français, autour du problème de la souffrance. Le contraste entre les deux femmes dans une loge de théâtre et Mère Marie-Yvonne dans sa cellule avec sa table et son crucifix, aurait-il paru toujours trop troublant ? Pour qui et pourquoi ? Les circonstances entourant la prise de voile en 1932 hanteraient-elles toujours les mémoires ?

En un sens, oui. On maintient bien l'ordre des choses. Car même Mère Marie-Yvonne reste définitivement la comédienne qui est entrée au couvent. Le titre de sa notice nécrologique dans L'Aurore à sa mort en 1976 ? « L'ancienne actrice Yvonne Hautin en religion Mère Marie-Yvonne est décédée à Paris ».

\section{Notes}

1. Le Sarasota Herald-Tribune du 28 août 1934 relate le départ d'une autre jeune comédienne française, Maryse Wendling, devenue religieuse missionnaire en Afrique, comme le troisième exemple d'une entrée en religion après Suzanne Delorme et Yvonne Hautin.

2. Paris, Champion, 2007. Je renvoie les lecteurs à mon article paru dans Histoires littéraires ( ${ }^{\circ} 25$, janvier-février-mars 2005) où je traite du cas d'Yvonne Hautin dans la perspective plus générale de la conversion d'actrices. L'article s'intitule « Actrices en religion : entre le Diable et le Bon Dieu ».

3. $\mathrm{N}^{\circ}$ 254, mai 2001. L'auteur de ce livre est Anne Martin-Fugier.

4. Nous nous appuyons essentiellement sur le recueil de presse contenue dans la boîte consacrée à Yvonne Hautin dans les archives de la Comédie-Française.

5. En fait, la presse provinciale reproduisait, parfois mot à mot, les articles de la presse parisienne. Dans un certain nombre de cas, nous avons pourtant bien voulu montrer l'intérêt qu'a suscité la prise de voile en dehors de la capitale. Le reportage de 1932 est souvent accompagné d'illustrations photographiques. Pour ce qui suit, nous ne donnons dans le texte, sauf indication contraire, que la source, car la plupart des articles de presse concernant la prise de voile renvoient au 18 juin 1932.

6. Paris, Castermann, 1965.

7. Paris, Desclée de Brouwer, coll. « Problèmes d'aujourd'hui », 1969.

8. Ce programme est accessible sur le site www.ina.fr/video/CP85102263, consulté le 6 octobre 2017. 